Supporting Information for:

\title{
Sub-Picosecond Photoinduced Hole Transfer from a CdS Quantum Dot to a Molecular Acceptor Bound Through an Exciton-Delocalizing Ligand
}

Shichen Lian, David J. Weinberg, Rachel D. Harris, Mohamad S. Kodaimati, and Emily A. Weiss*

Department of Chemistry, Northwestern University, 2145 Sheridan Rd., Evanston, IL 602083113

*corresponding author. Email: e-weiss@northwestern.edu

Sizing of CdS QDs through Ground State Absorption Spectroscopy. We determined the size of the synthesized CdS QDs (and their respective extinction coefficient) from the position of the first excitonic peak $(\sim 420 \mathrm{~nm})$ using the calibration curve published by $\mathrm{Yu}$ et al. ${ }^{1}$ All concentrations of QDs were calculated from the absorbance of QDs at $420 \mathrm{~nm}$ before ligand exchange.

\section{Determination of the HOMO Levels of PTC-PTZ and BA-PTZ by Cyclic Voltammetry.} We estimate the HOMO energy levels of the PTZ moiety when functionalized with either PTC or BA through cyclic voltammetry. The standard potentials for the ferrocene/ferrocenium couple is +0.34 V vs. SCE. ${ }^{2}$ The cyclic voltammogram of PTC-PTZ (Figure S1, black) shows two oxidation waves at $-0.08 \mathrm{~V}$ (vs. SCE) and $0.68 \mathrm{~V}$ (vs. SCE), which can be attributed to oxidizing the PTC and the PTZ moiety, respectively. We then convert the potential to energy relative to vacuum by adding $4.7 \mathrm{~V}$ to the value vs. SCE, which is $-5.38 \mathrm{eV}$ vs. vaccum. ${ }^{3}$ The CV scan of $1 \mathrm{mM}$ of BAPTZ (Figure S1B) shows that the HOMO of PTZ when attached with BA is $0.69 \mathrm{~V}$ (vs. SCE), which is $-5.39 \mathrm{eV}$ in energy vs. vacuum.
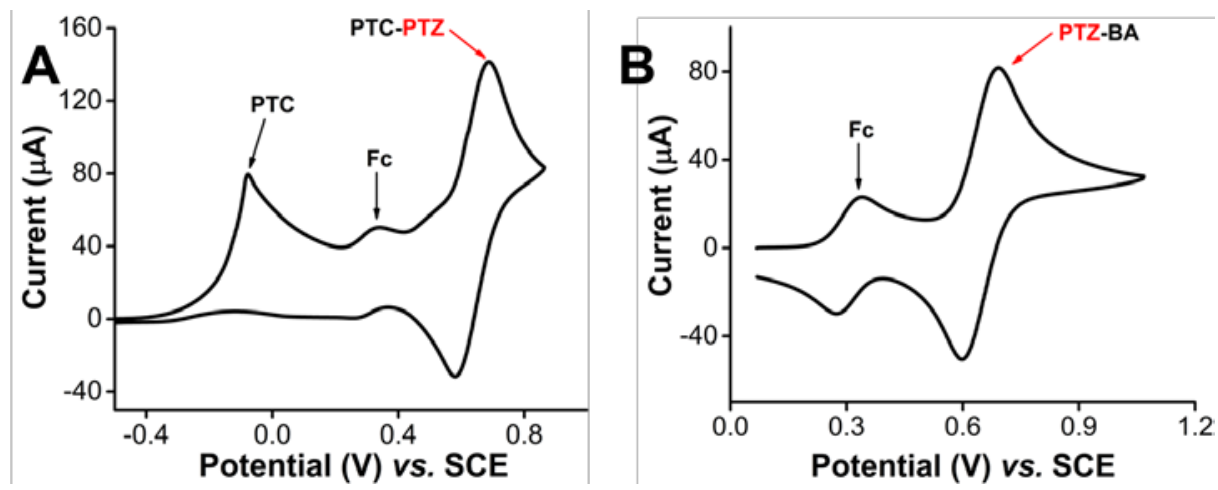

Figure S1. Cyclic voltammogram of $1 \mathrm{mM}$ (A) PTC-PTZ (B) BA-PTZ with $50 \mathrm{mM}$ tetrabutylammonium hexafluorophosphate in acetonitrile with added ferrocene as an internal standard in a three-electrode cell: a glassy carbon working electrode (apparent area of $28 \mathrm{~mm}^{2}$ ), a silver wire reference electrode, and a platinum wire counter electrode. 
Proton Assignment of the NMR Spectrum of Figure 3B in the Main Text. Proton assignment of the NMR spectra used in the discussion in the main text (Figure S2) is based on our previous study ${ }^{4}$ and the reference spectra of phenothiazine acquired in $\mathrm{d}_{6}$-benzene. The doublet peaks $\mathrm{C}$ and $\mathrm{A}$ correspond to two protons on the PTZ moiety $\left(\mathrm{H}_{\mathrm{C}}\right)$ and two protons $\left(\mathrm{H}_{\mathrm{A}}\right)$ on the PTC moiety, respectively, within the PTC-PTZ molecule. Doublet peaks D and B are identified as two protons $\left(\mathrm{H}_{\mathrm{D}}\right)$ on the PTZ moiety and two protons $\left(\mathrm{H}_{\mathrm{B}}\right)$ on the PTC moiety respectively within PTZ-Ph- $\mathrm{NH}_{2}$ based on the reference spectrum of $\mathrm{PTZ}-\mathrm{Ph}-\mathrm{NH}_{2}$ acquired in $\mathrm{d}_{6}$-benzene. Doublet peak E corresponds to two protons on the PTZ moiety $\left(\mathrm{H}_{\mathrm{E}}\right)$ of PTZ-Ph-TU, as discussed below.
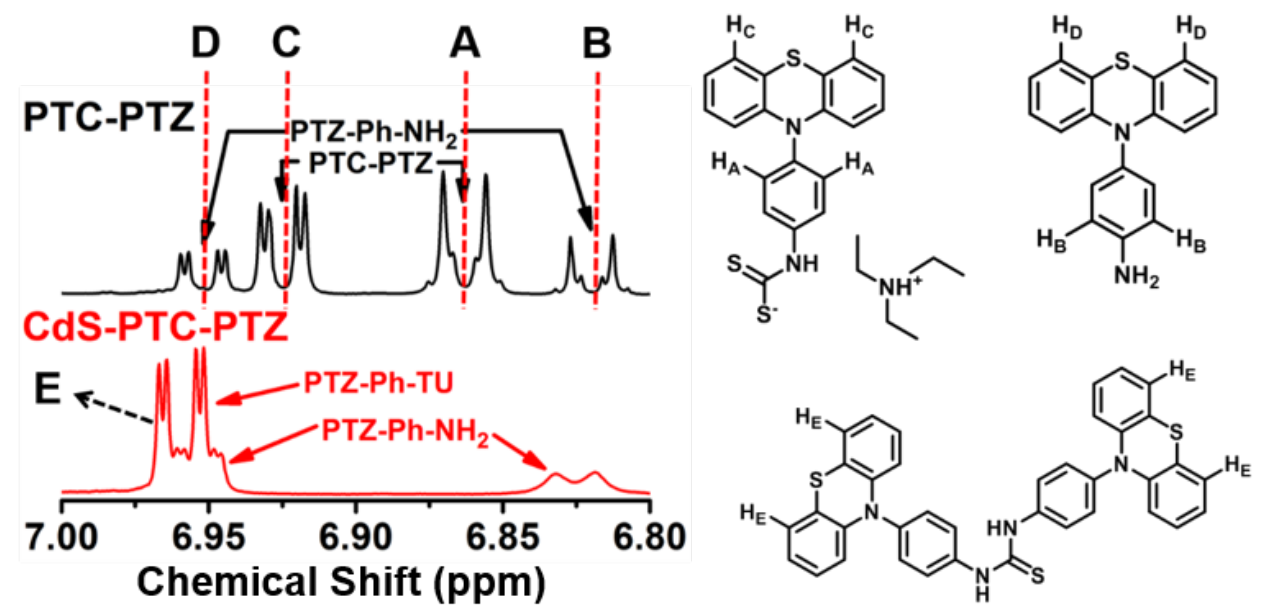

Figure S2. Proton assignment of the selected aromatic region of NMR spectra used in the discussion in the main text.

NMR Spectra of QD-PTZ Conjugates. In the presence of QDs, all proton NMR peaks of bound PTC-PTZ broaden into the baseline and the unbound PTC-PTZ decomposes into PTZ-Ph$\mathrm{NH}_{2}$ and PTZ-Ph-TU, Figure S3. The peaks within the gray shaded area are the signals from PTCPTZ molecules. The triplet and doublet peaks at $\sim 6.6 \mathrm{ppm}$ in the top spectrum correspond to the PTC-PTZ degradation products, PTZ-Ph-NH2 and PTZ-Ph-TU. 


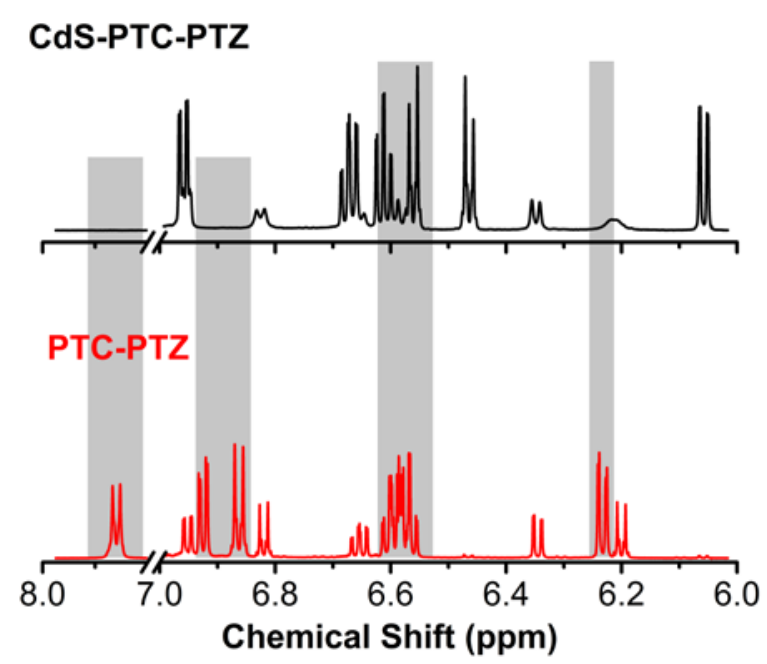

Figure S3. ${ }^{1} \mathrm{H}$ NMR spectra of PTC-PTZ with (top) and without (bottom) CdS QDs.

PTC-PTZ degrades to PTZ-Ph-TU and PTZ-Ph-NH 2 in the presence of QDs. Figure S3 shows that the minor impurities within CdS-PTC-PTZ at $~ 6.8$ ppm (doublet), 6.65 (triplet), 6.58 ppm (triplet), 6.35 ppm (doublet), and 6.2 ppm (doublet) all correspond to PTZ-Ph-NH2. The rest of the peaks overlay with the proton signals of PTC-PTZ mixed with $\mathrm{Cd}(\mathrm{OA})_{2}$ complexes. MALDI-TOF data show that the dominant species in these mixtures (Cd(OA) $)_{2}+$ PTC-PTZ) is PTCPh-TU. We therefore conclude that, when PTC-PTZ ligands are not bound to the surfaces of QDs, they decompose into $\mathrm{PTZ}-\mathrm{Ph}-\mathrm{NH}_{2}$ or undergo various reactions with $\mathrm{Cd}(\mathrm{OA})_{2}$ to form $\mathrm{PTZ}-\mathrm{Ph}$ TU, which is consistent with our previous study. ${ }^{4}$

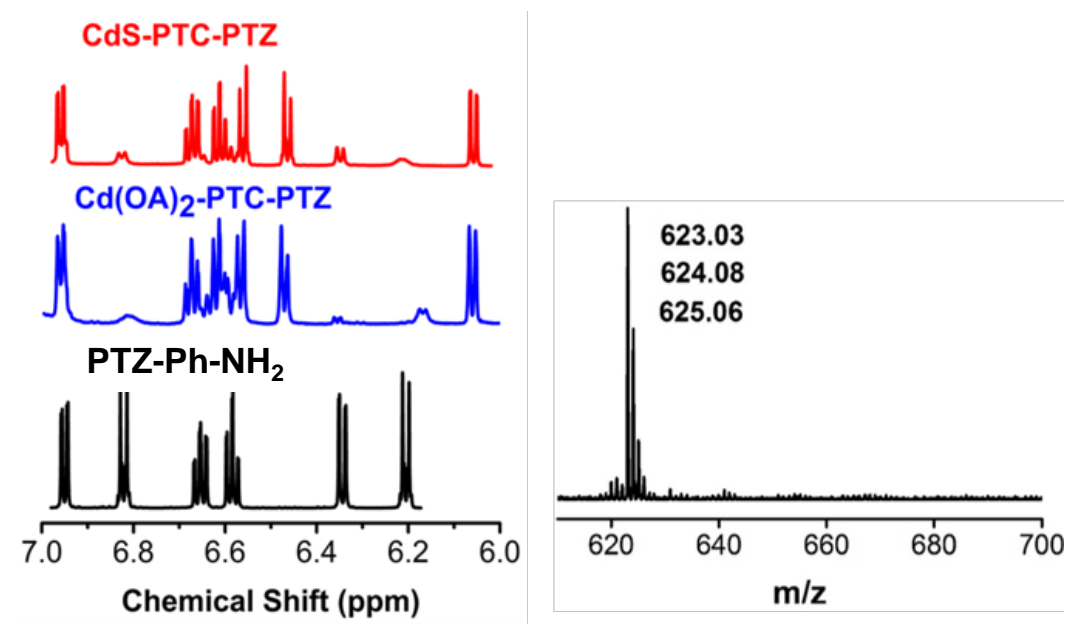

Figure S4. (A) ${ }^{1} \mathrm{H}$ NMR spectra of CdS-PTC-PTZ, PTC-PTZ with Cd(OA) 2 complexes, and An-PTZ. (B) MALDI-TOF mass spectrum $[\mathrm{M}-\mathrm{H}]^{+}$of PTC-PTZ mixed with $\mathrm{Cd}(\mathrm{OA})_{2}$. Calculated peaks: 622.13 (100.0\%), 623.14 (40.0\%), 624.13 (13.6\%), 624.14 (7.8\%), 625.13 (5.4\%), 623.13 (2.4\%), 623.13 (1.5\%), 626.13 (1.1\%) 
Determination of Ligand Quantification Error via NMR Calibration Curves. We estimate the error of our NMR experiments through a construction of a calibration curve (Figure S5), which we define as the discrepancy between the number of added phenyl rings associated with aromatic PTC species and the number of detected phenyl rings. We prepared six samples with different amounts of added PTC-PTZ molecules in mixtures with QDs. Due to the PTZ-Ph- $\mathrm{NH}_{2}$ impurity, it is unknown precisely how many equivalents of PTC-PTZ molecules are added. We therefore prepared another six samples with the same amount of added molecules (from the same stock solution to ensure the homogeneity) without QDs and used the integrated proton NMR signals of the phenyl ring that attached to the PTZ moiety to calculate how many phenyl rings are added, which is the $\mathrm{x}$-axis in Figure S5. We identified each degradation product and measured the corresponding \% mass for the six samples with QDs and obtained the number of detected phenyl rings, which is the y-axis in Figure S5. We used the quantity (\# of displaced OA/2) as the number of bound PTC-PTZ, as explained in the main text. We then plotted the number of detected phenyl rings against the number of added phenyl rings and fit the data with a linear function with forced intercept at $(0,0)$. We use the deviation $(13 \%)$ from the ideal response line $(1: 1)$ as the error for our NMR measurements.

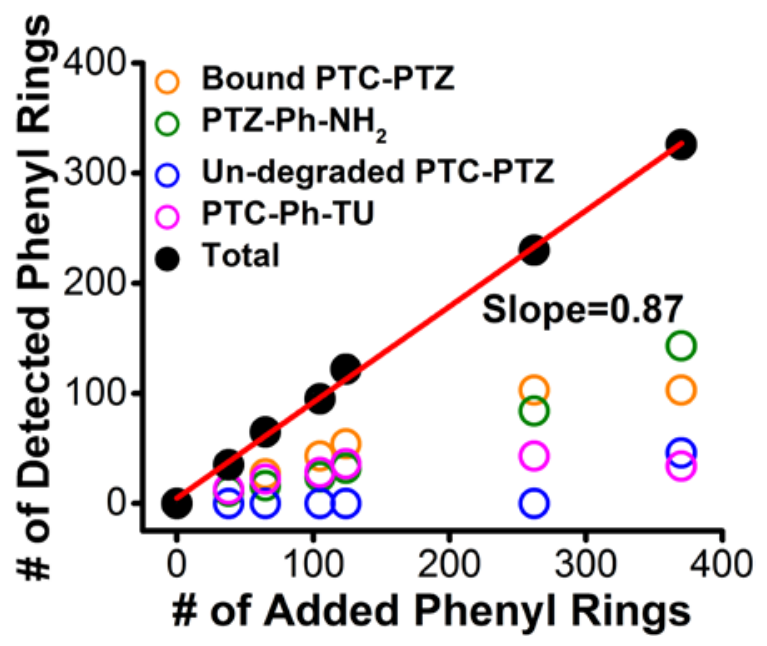

Figure S5. Plot of the measured concentration of phenyl rings attached to the PTZ moiety versus the concentration of added phenyl rings, as determined by integrated intensity of one proton on the phenyl ring. 
Photoluminescence of CdS-BA and CdS-PTC. Figure S6 shows the PL spectra of CdS QDs treated with either BA or PTC with no attached PTZ moieties. Treatment with 55 eq. of BA per QD enhances the PL of CdS QDs by a factor of 2. Treatment with 110 eq. of C6-PTC (4-hexylphenyldithiocarbamate) per QD enhances the PL of QDs by 10\%. Adding $\mathrm{Cd}^{2+}$ into the system is necessary to precipitate the degradation products of C6PTC, 4-hexylaniline, 4hexylphenylisothiocyanate, and N,N'-bis(4-hexylphenyl)-thiourea, which, as we showed in detail in a previous study, quench the PL of the QDs. ${ }^{4,5}$

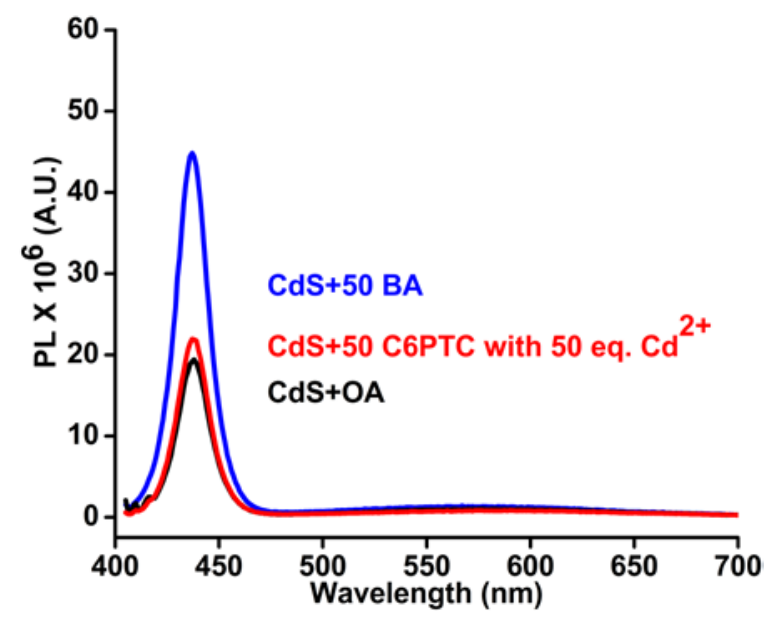

Figure S6. Overlaid photoluminescence spectra of $10 \mathrm{uM}$ of CdS QDs coated with OA (black), treated with 55 eq. of BA (blue) and treated with 110 eq. of C6PTC per QD (red), excited at $400 \mathrm{~nm}$.

\section{Raw TA spectra of CdS-PTC-PTZ and CdS-BA-PTZ, Basis Spectra of the QD Excited} State and PTZ $^{+\bullet}$, and the Spectral Deconvolution Procedure. As discussed in the main text, the raw TA spectra of CdS-PTC-PTZ and CdS-BA-PTZ, excited at $420 \mathrm{~nm}$, have two overlapping features in the visible region: i) the absorption of $\mathrm{PTZ}^{+}$, which peaks at $520 \mathrm{~nm}$, and ii) an excited state absorption feature of QDs (denoted the "shelf”) from 480 nm to 700 nm (Figure S7). The shape of the shelf feature does not evolve in time, but its amplitude does. In order to isolate the kinetics of $\mathrm{PTZ}^{+\bullet}$, we deconvoluted the raw transient absorption data as a linear combination of the basis spectra of $\mathrm{PTZ}^{+\bullet}$ and shelf.

In order to isolate the basis spectrum of the QD shelf in the CdS-PTC-PTZ samples, we first took a TA spectrum from the CdS-PTC control sample (without the PTZ moiety, but with similar PTC coverage, spectrum A) and used spectrum A as the basis spectrum for shelf in CdS-PTC-PTZ. 
Next, in order to obtain the basis spectrum of $\mathrm{PTZ}^{+\bullet}$, we extracted a TA spectrum for CdS-PTCPTZ at $\sim 2.9$ ns (spectrum B), which is dominated by the radical cation. We then scaled spectrum A such that the optical density between $620 \mathrm{~nm}$ to $650 \mathrm{~nm}$ matched that in spectrum $\mathrm{B}$, and then subtracted the scaled spectrum A from spectrum B to eliminate all contribution of the QD shelf and isolate the basis spectrum of $\mathrm{PTZ}^{+\bullet}$. We used the same method to get the basis spectra for CdSBA-PTZ.

The deconvolution of the raw TA dataset was performed by a linear combination of these two basis spectra using a MATLAB software. ${ }^{6}$ The output from this method is a species-associated kinetic dataset, which contains respective contributions of these two basis species to the overall raw TA spectrum at each time point.

In order to cross-check the accuracy of this method (i.e. verify that the TA data can be fit as a weighted sum of two basis spectra), we compared the raw kinetic trace at $630 \mathrm{~nm}$ (only contains the shelf feature) from CdS-PTC-PTZ or CdS-BA-PTZ spectra with the kinetic trace corresponding to the shelf feature generated from the deconvolution procedure for the same sample. The agreement between these two kinetic traces (Figure S8) indicates that the deconvoluted species-associated kinetic data are representative of the transient dynamics of the shelf and $\mathrm{PTZ}^{+\bullet}$ species. Basis spectra used for this procedure and raw TA spectra for the rest of QD samples with different coverages are in Figure S9. 

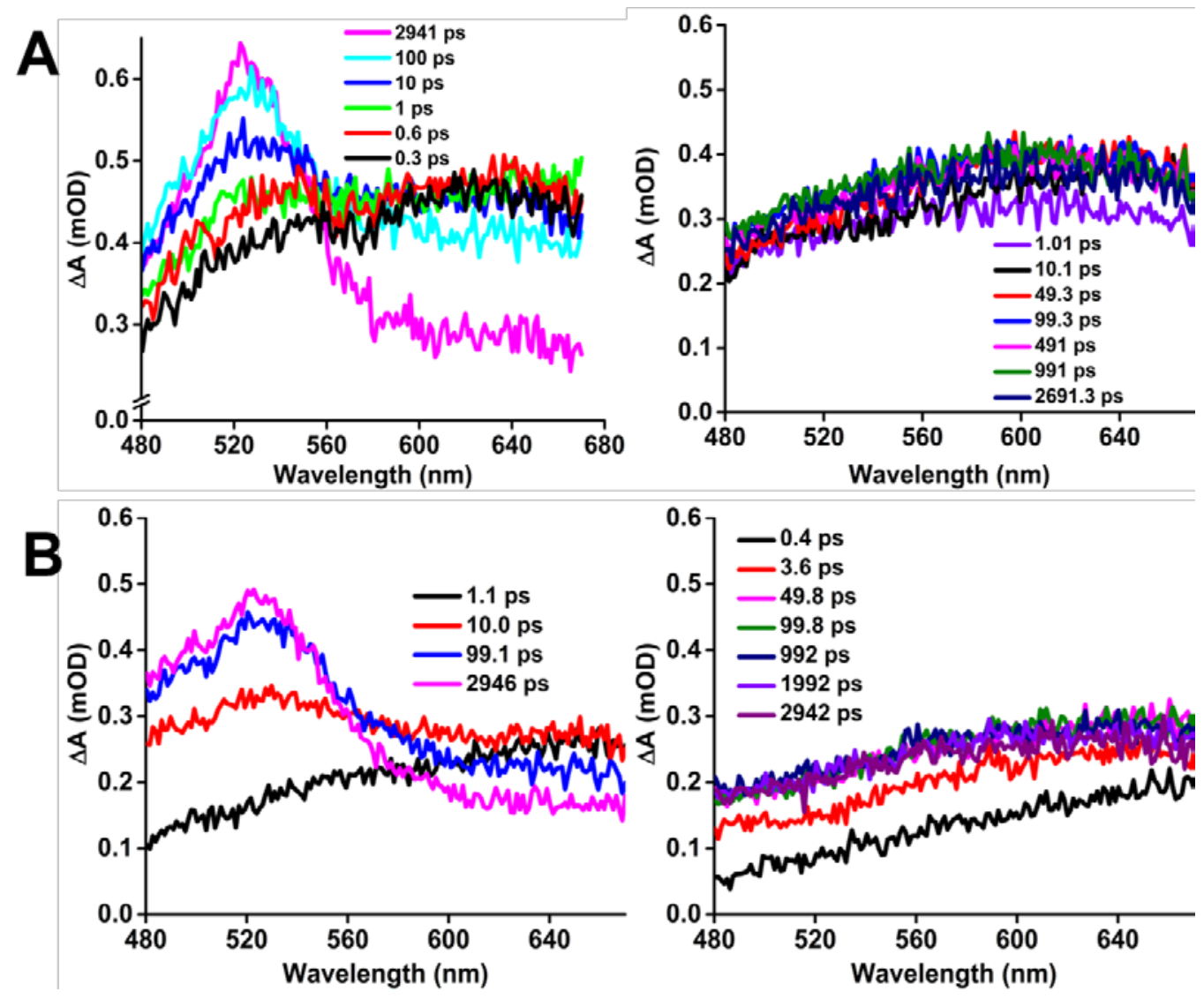

Figure S7. Raw TA spectra (before deconvolution) of $10 \mu \mathrm{M}$ of CdS QDs with (A) 49 bound PTC-PTZ (left) and 49 bound C6PTC (right), (B) 48 bound BA-PTZ and 50 bound BA (right), at various times after excitation at $420 \mathrm{~nm}$.
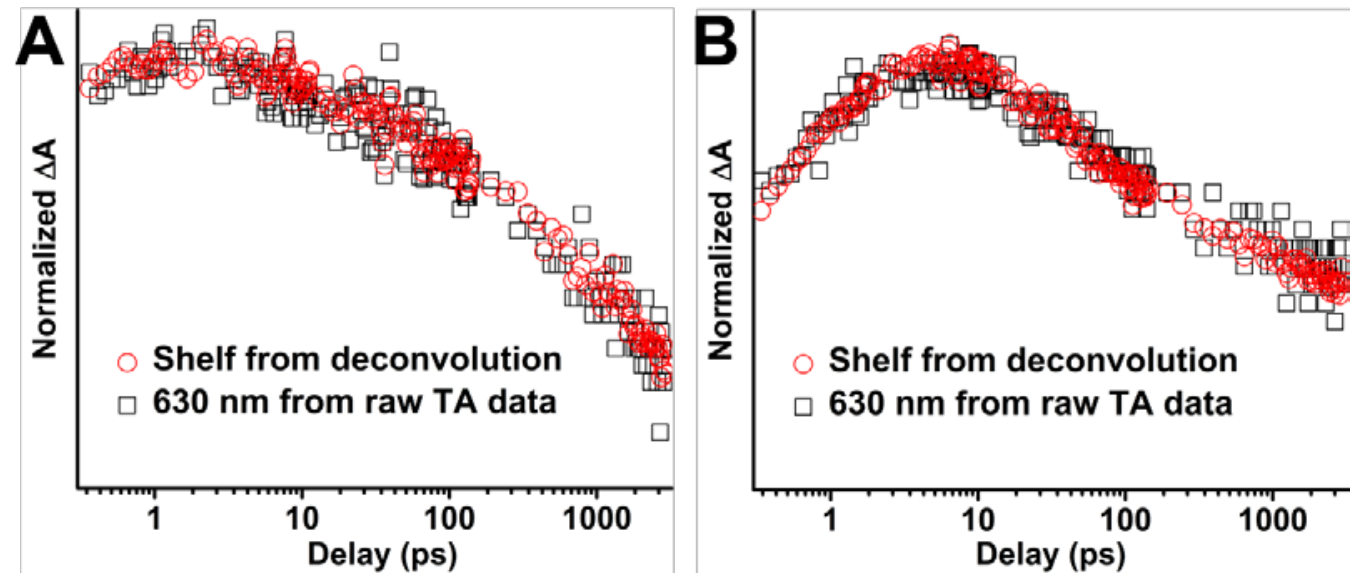

Figure S8. Overlaid normalized TA kinetic traces of the "shelf" obtained from the deconvolution procedure (red) and extracted at $630 \mathrm{~nm}$ from the raw TA data at $630 \mathrm{~nm}$ (black) of CdS QDs with (A) 49 bound PTC-PTZ and (B) 48 bound BA-PTZ. The spectrum before $300 \mathrm{fs}$ is truncated for clarity as it contains the nonlinear response from solvent. 

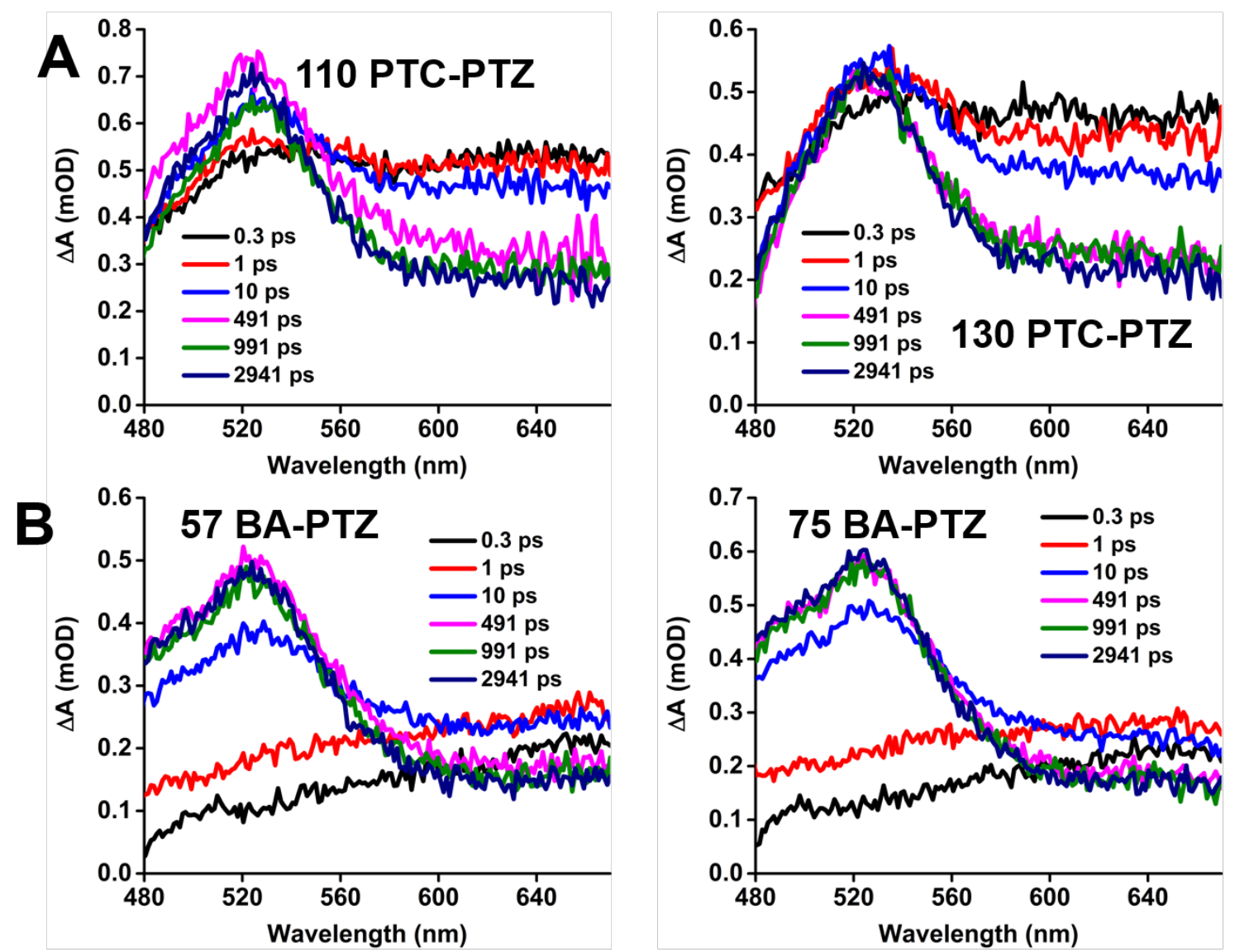

C
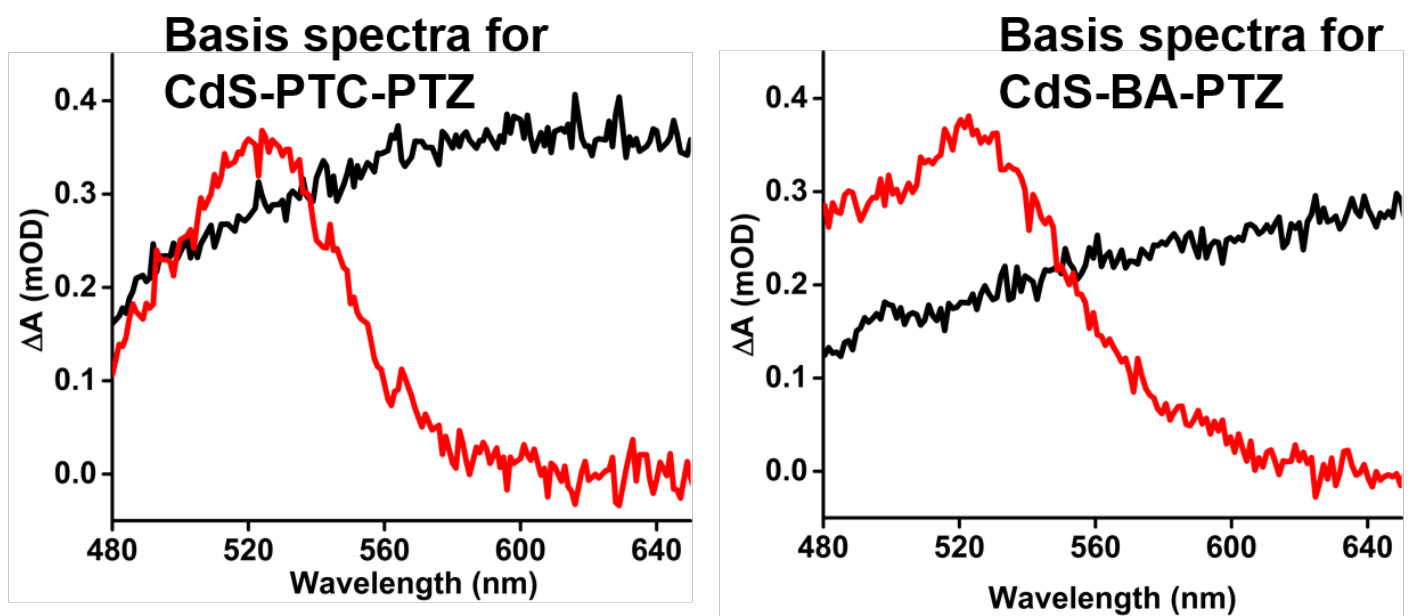

Figure S9. Raw TA spectra of CdS QDs with different surface coverages of (A) PTC-PTZ and (B) BA-PTZ. (C) Left: basis spectra of $\mathrm{PTZ}^{+\bullet}$ (red) and shelf (black), obtained from reconstructing the TA data of CdS-PTC-PTZ samples. Right: basis spectra of $\mathrm{PTZ}^{+\bullet}$ (red) and shelf (black), obtained from reconstructing the TA data of CdS-BA-PTZ samples. 


\section{Assignment of Hole Trapping, Trapped Hole Transfer, and Valence Band Hole Transfer.}

Table S1 summarizes the time constants for the fits of i) the raw kinetic trace at a probe wavelength of $630 \mathrm{~nm}$, ii) the raw kinetic trace at a probe wavelength of $1300 \mathrm{~nm}$, and iii) the deconvoluted $\mathrm{PTZ}^{+\bullet}$ trace for CdS QDs with 48 bound BA-PTZ. As explained in the main text, kinetics at 630 $\mathrm{nm}$ correspond primarily to dynamics of the trapped hole and kinetics at $1300 \mathrm{~nm}$ correspond primarily to the dynamics of the valence band hole. For the CdS-BA-PTZ system, we therefore assign $\tau_{\text {trap }}$ (a rise component at $630 \mathrm{~nm}$ and a decay component at $1300 \mathrm{~nm}$ ) to be the depopulation of the valence band holes by transfer to trap states. $\tau_{1}$ is transfer of valence band holes from QDs to PTZ because this time constant is present both at $520 \mathrm{~nm}$ (rise) and $1300 \mathrm{~nm}$ (decay). $\tau_{2}$ and $\tau_{3}$ are transfer of trapped holes from trap states to the HOMO of PTZ because these two constants are present at both $520 \mathrm{~nm}$ (rise) and $630 \mathrm{~nm}$ (decay). Plot of these kinetic traces with fitting functions are in Figure S10.
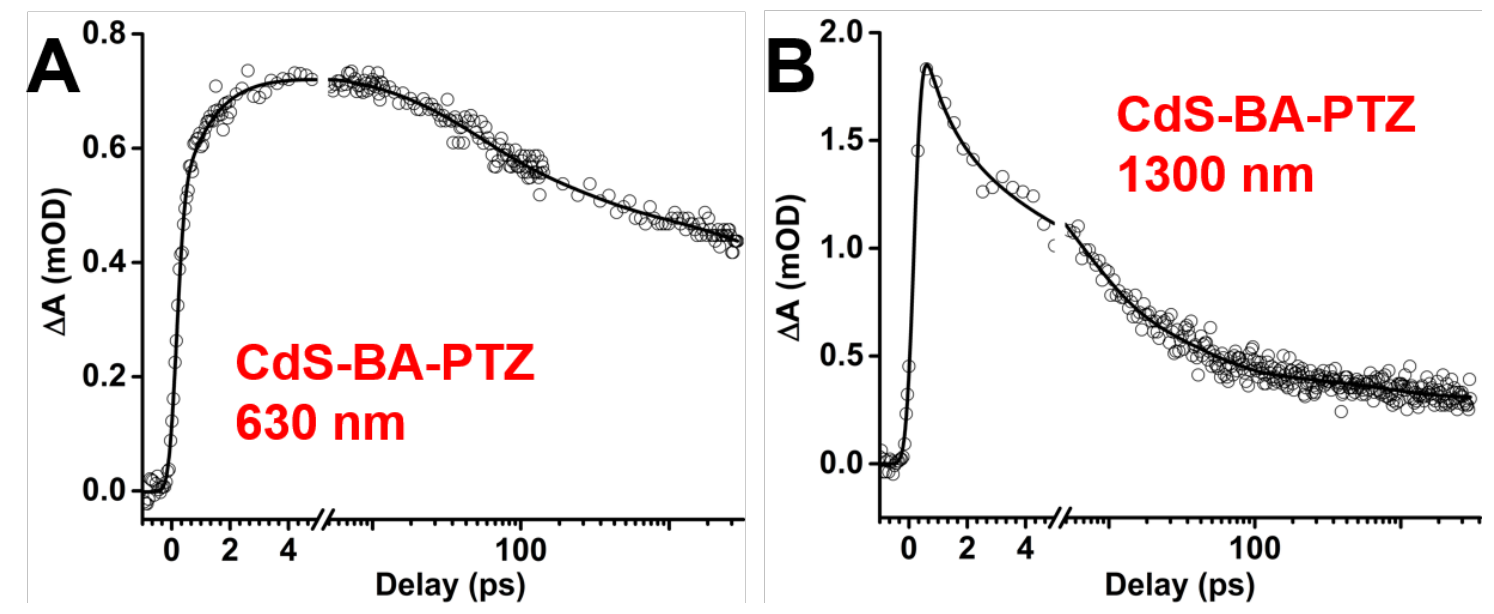

Figure S10. Kinetic traces extracted at (A) $630 \mathrm{~nm}$ and (B) $1300 \mathrm{~nm}$ for $10 \mu \mathrm{M}$ of CdS QDs bound with 48 BA-PTZ. 
Table S1. Time Constants for PTZ ${ }^{+\bullet}$ and Excited-State Decay of CdS QDs with 48 bound BA-PTZ, Probed at Three Different Wavelengths

\begin{tabular}{|c|c|c|c|c|c|c|c|}
\hline $\begin{array}{l}\text { CdS-BA- } \\
\text { PTZ }\end{array}$ & $\tau_{\text {trap }}, \mathrm{ps}$ & $\tau_{1}, \mathrm{ps}$ & $\tau_{2}, \mathrm{ps}$ & $\tau_{3}, \mathrm{ps}$ & $\tau_{4}, \mathrm{ps}^{a}$ & $\tau_{5}, \mathrm{ps}$ & $\tau_{6}, \mathrm{ps}$ \\
\hline Assignment & $\begin{array}{c}\mathrm{h}^{+} \\
\text {trapping }\end{array}$ & $\begin{array}{l}\mathrm{VB} \mathrm{h}^{+} \\
\text {transfer }\end{array}$ & $\begin{array}{c}\text { trapped } \\
\mathrm{h}^{+} \\
\text {transfer }\end{array}$ & $\begin{array}{l}\text { trapped } \\
\mathrm{h}^{+} \\
\text {transfer }\end{array}$ & $\begin{array}{c}\text { possible } \\
\text { CB e- }\end{array}$ & $\begin{array}{c}\text { possible } \\
\text { CB e- }\end{array}$ & $\begin{array}{c}\text { exciton } \\
\text { decay }\end{array}$ \\
\hline $\begin{array}{c}\text { deconvolved } \\
\text { PTZ }^{+\bullet} \\
\end{array}$ & & $5.5 \pm 0.5$ & $34 \pm 6$ & $350 \pm 70$ & & & \\
\hline $\begin{array}{c}630 \mathrm{~nm} \\
(\text { mostly } \\
\left.\text { trapped } h^{+}\right)\end{array}$ & $1.1 \pm 0.1$ & & $40 \pm 10$ & $200 \pm 100$ & & & $>3000$ \\
\hline $\begin{array}{c}1300 \mathrm{~nm} \\
(\text { mostly VB } \\
\left.\mathrm{h}^{+}\right) \\
\end{array}$ & $0.8 \pm 0.4$ & $6 \pm 1$ & & & $38 \pm 7$ & $800 \pm 200$ & \\
\hline
\end{tabular}

${ }^{a}$ This component is not likely responsible for excitonic hole transfer because hole trapping is on the single picosecond time scale and the time constant for this component is almost 40 times bigger.

We assign hole trapping, trapped hole transfer, and valence band hole transfer components within CdS-PTC-PTZ based on a similar reasoning. Although we cannot reliably detect an instrument-limited decay at $1300 \mathrm{~nm}$, we assign the ultrafast oxidation of PTZ ( $\tau_{1}$ in the main text) to hole transfer from the valence band of the QDs to PTZ through PTC, because (i) we show directly that the fastest hole transfer time constant is transfer of an excitonic hole to PTC in the CdS-BA-PTZ system, (ii) we can definitively assign the longer hole transfer time constant $\tau_{2}$ to oxidation of PTZ by a trapped hole, as described below, and (iii) it does not make sense that transfer of a trapped hole to PTZ would take place on a sub-picosecond timescale given that it must occur in two steps (hole trapping and trapped hole transfer). We assign component $\tau_{2}$ to trapped hole transfer to PTZ as these two time constants are present at $630 \mathrm{~nm}$ in all three samples (Table S2). We assign $\tau_{3}$ and $\tau_{4}$ to hole transfer within $\mathrm{PTZ}-\mathrm{Ph}-\mathrm{NH}_{2}$ because these two time constants match with the ones of the control experiment where we added PTZ-Ph- $\mathrm{NH}_{2}$ directly into oleate-capped QDs. These two time constants depend on the number of PTZ-Ph-NH 2 transiently bind to the surface of QDs, as described later in this document (Table S4). Table S1S3 summarize all fitting parameters of the kinetic traces at relevant wavelengths (plotted in Figure S9 and S10), for CdS-BA-PTZ and CdS-PTC-PTZ samples with different surface coverages of BA-PTZ and PTC-PTZ. We use eq S1,

$$
y=I R F \otimes \sum_{1}^{n} A_{n} e^{\frac{-\left(t-t_{0}\right)}{\tau_{n}}}
$$


a sum of exponential functions convoluted with an instrument response function (in this case, a Gaussian pulse) to fit all the TA kinetic traces. We choose $n$ to be the minimum integer to obtain a satisfactory fit, defined as a fit that results in symmetric scattering of residuals around a zero line at all delay times.

Table S2. Time Constants for PTZ ${ }^{+\bullet}$ and Excited-State Decay of CdS QDs with Different Coverages of PTC-PTZ at Two Different Wavelengths

\begin{tabular}{|c|c|c|c|c|c|c|c|}
\hline & $\begin{array}{l}\text { Wavelength } \\
\text { (nm) }\end{array}$ & $\begin{array}{c}\tau_{\text {trap, }} \text { ps } \\
\mathrm{h}^{+} \\
\text {trapping }\end{array}$ & $\begin{array}{c}\tau_{2}, \mathrm{ps} \\
\text { tapped } \\
\mathrm{h}^{+} \\
\text {transfer }\end{array}$ & $\begin{array}{c}\tau_{3}, \mathrm{ps} \\
\mathrm{h}^{+} \\
\text {transfer } \\
\text { to PTZ- } \\
\text { Ph-NH}\end{array}$ & $\begin{array}{c}\tau_{4}, \mathrm{ps} \\
\mathrm{h}^{+} \\
\text {transfer } \\
\text { to PTZ- } \\
\text { Ph-NH2 }\end{array}$ & $\tau_{5}, \mathrm{ps}^{a}$ & $\begin{array}{c}\tau_{6}, \mathrm{ps} \\
\text { exciton } \\
\text { decay }\end{array}$ \\
\hline \multirow{2}{*}{$\begin{array}{c}49 \\
\text { PTC- } \\
\text { PTZ }\end{array}$} & $\begin{array}{c}\text { deconvolved } \\
\text { PTZ }^{+}\end{array}$ & - & $5.1 \pm 0.7$ & $90 \pm 10$ & $\begin{array}{c}2000 \pm 10 \\
00\end{array}$ & - & \\
\hline & 630 & $<0.3$ & $6 \pm 2$ & - & - & $160 \pm 30$ & $>3000$ \\
\hline \multirow{2}{*}{$\begin{array}{c}110 \\
\text { PTC- } \\
\text { PTZ }\end{array}$} & $\begin{array}{l}\text { deconvolved } \\
\text { PTZ }^{+\bullet}\end{array}$ & - & $1.6 \pm 0.4$ & $24 \pm 3$ & $700 \pm 100$ & - & \\
\hline & 630 & $<0.3$ & $6 \pm 1$ & - & - & $110 \pm 10$ & $>3000$ \\
\hline \multirow{2}{*}{$\begin{array}{c}130 \\
\text { PTC- } \\
\text { PTZ }\end{array}$} & $\begin{array}{c}\text { deconvolved } \\
\text { PTZ }^{+\bullet}\end{array}$ & - & $2.6 \pm 0.6$ & $33 \pm 4$ & - & - & - \\
\hline & 630 & $<0.3$ & $2.9 \pm 0.4$ & - & - & $80 \pm 10$ & $>3000$ \\
\hline
\end{tabular}

${ }^{a}$ We do not assign this component because it is probably a convolution of conduction band electron transitions and hole transfer to $\mathrm{PTZ}-\mathrm{Ph}-\mathrm{NH}_{2}$. 
Table S3. Time Constants for PTZ ${ }^{+\bullet}$ and Excited-State Decay of CdS QDs with Different Coverages of BA-PTZ at Two Different Wavelengths

\begin{tabular}{|c|c|c|c|c|c|c|}
\hline $\begin{array}{c}\text { Coverage } \\
\text { per QD }\end{array}$ & $\begin{array}{c}\text { Wavelength } \\
(\mathrm{nm})\end{array}$ & $\begin{array}{c}\tau_{\text {trap, }} \mathrm{ps} \\
\mathrm{h}^{+} \\
\text {trapping }\end{array}$ & $\begin{array}{c}\tau_{1}, \mathrm{ps} \\
\mathrm{VB} \mathrm{h}^{+} \\
\text {transfer }\end{array}$ & $\begin{array}{c}\tau_{2}, \mathrm{ps} \\
\text { trapped } \mathrm{h}^{+} \\
\text {transfer }\end{array}$ & $\begin{array}{c}\tau_{3}, \mathrm{ps} \\
\text { trapped } \mathrm{h}^{+} \\
\text {transfer }\end{array}$ & $\begin{array}{c}\tau_{4} \text {, ps } \\
\text { exciton decay }\end{array}$ \\
\hline $\begin{array}{c}\text { 48 BA- } \\
\text { PTZ }\end{array}$ & $\begin{array}{c}\text { deconvolved } \\
\mathrm{PTZ}^{+}\end{array}$ & - & $5.5 \pm 0.5$ & $34 \pm 6$ & $350 \pm 70$ & - \\
\cline { 2 - 7 } & 630 & $1.1 \pm 0.1$ & - & $40 \pm 10$ & $200 \pm 100$ & $>3000$ \\
\hline $\begin{array}{c}\text { 57 BA- } \\
\text { PTZ }\end{array}$ & $\begin{array}{c}\text { deconvolved } \\
\mathrm{PTZ}^{+}\end{array}$ & - & $2.6 \pm 0.8$ & $9 \pm 3$ & $90 \pm 10$ & - \\
\cline { 2 - 7 } & 630 & $0.4 \pm 0.03$ & - & $12 \pm 4$ & $80 \pm 30$ & $>3000$ \\
\hline $\begin{array}{c}\text { 75 BA- } \\
\text { PTZ }\end{array}$ & $\begin{array}{c}\text { deconvolved } \\
\mathrm{PTZ}^{+\bullet}\end{array}$ & - & $2.8 \pm 0.2$ & $17 \pm 2$ & $300 \pm 100$ & - \\
\cline { 2 - 7 } & 630 & $0.35 \pm 0.0$ & - & $14 \pm 2$ & $380 \pm 90$ & $>3000$ \\
\hline
\end{tabular}
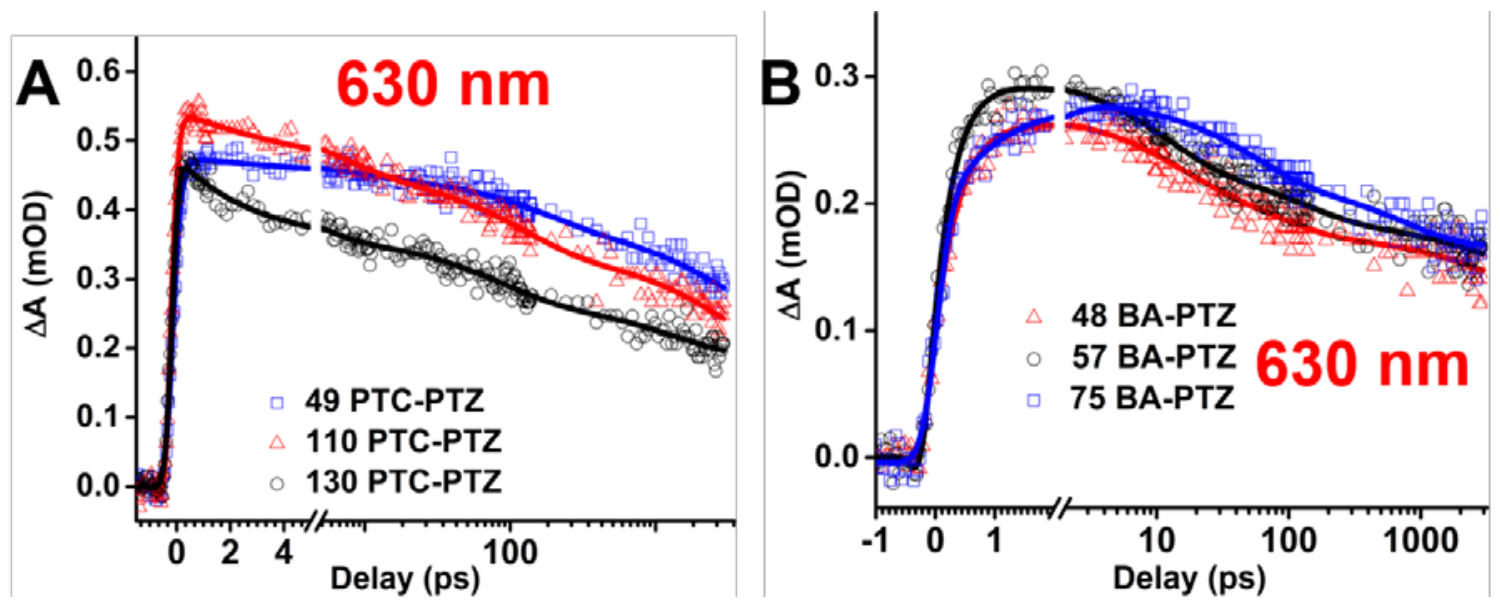

Figure S11. Kinetic traces extracted at $630 \mathrm{~nm}$ for $10 \mu \mathrm{M}$ of CdS QDs bound with various coverages of (A) PTC-PTZ and (B) BA-PTZ. Fitting parameters are summarized in Table S2 and S3. 


\section{Measurement of the Instrument Response for Femtosecond Transient Absorption.}

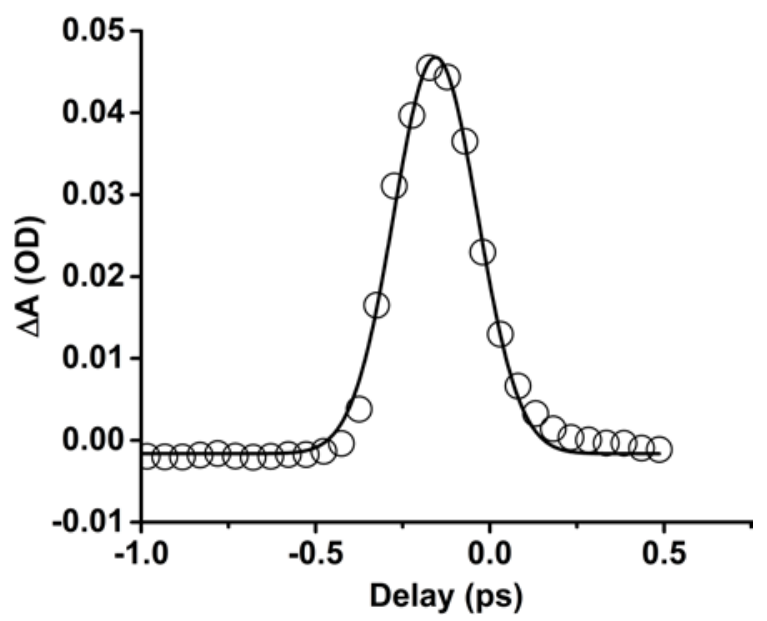

Figure S12. The instrument response function (IRF), given by the cross-correlation of the pump and probe, was measured by inducing the optical Kerr effect in a quartz cuvette containing $\mathrm{CCl}_{4}$ solution at the position of the sample. The full width at half maximum for the Gaussian fit is $280 \pm 10 \mathrm{fs}$.

Correction of TA Spectra. Our procedure to account for the effect of group velocity dispersion included: i) collection of the cross-correlation IRF with the use of carbon tetrachloride, ii) fitting of the IRF dispersion curve with a polynomial function, and iii) application of the IRF fit to correct the time-zero for all wavelengths of all samples. We identify the femtosecond coherent artifacts by comparing the TA spectra of pure solvent only with those of the QD sample (Figure S13). The TA spectrum of solvent was acquired under the same condition (power, spot size, etc.) as what we used for all other samples. Compared with Figure S13 (right), the small magnitude of solvent response and its time scale (within IRF) in Figure S13 (left) should not influence our interpretation of the TA signal of $\mathrm{PTZ}^{+\bullet}$ and our conclusions.
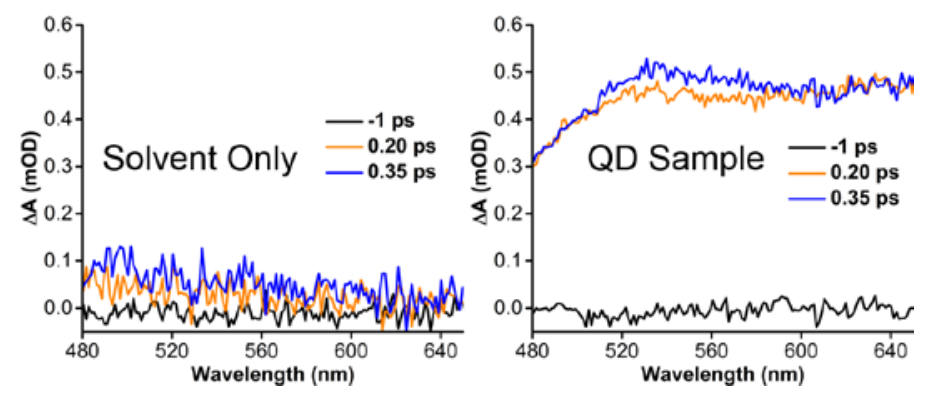

Figure S13. TA spectra of benzene- $d_{6}$ (left) and one representative QD sample used in this study (right) at various delay time. These two set of TA spectra were acquired at the same condition (pump wavelength, power, spot size, etc.). 
Calculation of the Expectation Value of the Excited-State QDs. We calculated the expectation value of the excited-state QDs based on a previous report. ${ }^{7}$ We measured the beam spot size at the position of the sample by translating a razor blade attached to a translation stage perpendicularly across the beam. A power meter was used to record the transmitted power in a function of translated distance (Figure S14). An error function was used to extract the beam spot size, which is defined as the FWHM of the Gaussian peak $(630 \pm 10 \mu \mathrm{m})$.

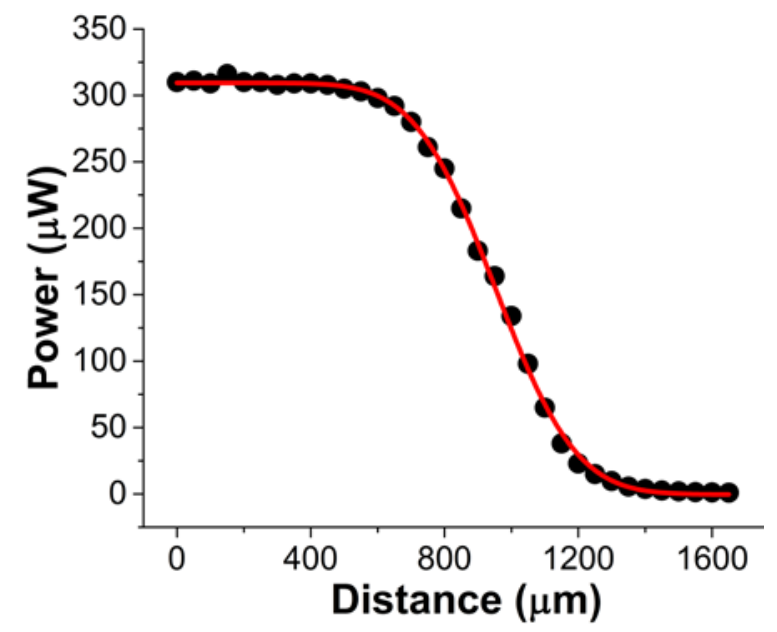

Figure S14. A plot of transmitted power vs. translated distance (black dots), which is used to fit an error function (red curve) to extract the beam spot size.

Photon flux is calculated based on the spot size and the expected excited-state population is calculated by using eq S2, which has accounted for attenuation. ${ }^{7}$

$$
\langle N\rangle=\frac{1000 I_{o}}{N_{a} \ell c}(1-\operatorname{Exp}[-\ln (10) \varepsilon \ell c]) \quad \mathrm{S} 2
$$

$<N>$ is the expectation value of excited-state QDs, $I_{o}$ is the photon flux, $N_{a}$ is Avagadro's number, $\ell$ is pathlength, $\varepsilon$ is the extinction coefficient of QDs at the pump wavelength, $c$ is the concentration of QDs. The calculated expectation value is $\sim 0.05$.

The lifetime of hole trapping increases with increasing coverage of PTC-PTZ. In order to investigate how the dithiocarbamate binding group influences the hole trapping dynamics when adsorbed to the QD, we added 4-hexylphenyldithiocarbamate (a PTC molecule with C6 alkyl chain at the para-position, with no attached PTZ) into $10 \mu \mathrm{M}$ QDs; the alkyl chain increases the solubility 
of the PTC-coated QDs in benzene, so that we can achieve higher coverage. As we discussed in the main text, the rise component of the 630-nm kinetic trace corresponds to hole trapping from the delocalized VB of the QD to a lattice or surface state. We show in Figure S12 that our instrument response function is $280 \mathrm{fs}$, as measured by the optical Kerr effect. Figure S13 shows that the lifetime of this component decreases from $~ 1$ ps to sub-picosecond (instrument-limited), with increasing coverage of C6PTC on the QD, which indicates that, with addition of dithiocarbamate molecules into QDs, the hole trapping process gets faster. Hole transfer to PTZ must therefore also get faster with increasing coverage of PTC-PTZ, in order to compete with the accelerated hole trapping.

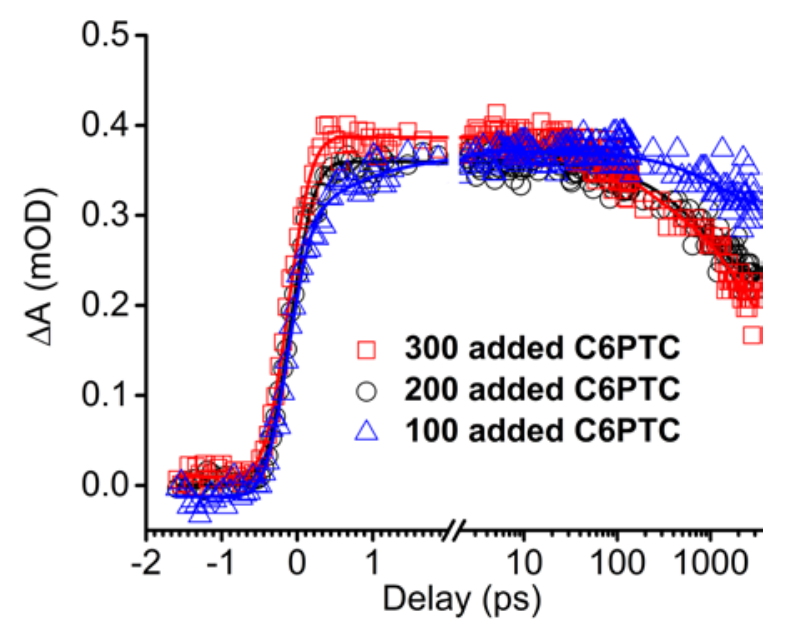

Figure S15. Overlaid kinetic traces extracted at $630 \mathrm{~nm}$ from the TA spectra of $10 \mathrm{uM}$ of CdS QDs with various amount of added 4-hexylphenyldithiocarbamate (C6PTC), after excitation at $420 \mathrm{~nm}$.

Hole Transfer Within CdS-NH2-Ph-PTZ. The broadened NMR peaks of PTZ-Ph-NH indicate that PTZ-Ph- $\mathrm{NH}_{2}$, which is a degradation product of PTC-PTZ, weakly binds to the QDs. When we added 1000 eq. of PTZ-Ph-NH timescale than that to BA-PTZ or PTC-PTZ, Table S4. We used the same deconvolution procedure described previously to obtain the kinetic corresponding to $\mathrm{PTZ}^{+\bullet}$ in this sample (Figure S14). We suspect that, with increasing amounts of added PTC-PTZ, the dithiocarbamate displaces $\mathrm{Cd}(\mathrm{OA})_{2}$ on the QD surface, making it easier for PTZ-Ph- $\mathrm{NH}_{2}$ molecules to access the surface of QDs; thus more An-PTZ molecules bind transiently to the QD with increasing coverage of PTC-PTZ, and 
the hole transfer occurs faster within 110 PTC-PTZ and 130 PTC-PTZ samples than within the 49 PTC-PTZ sample, Table S4.
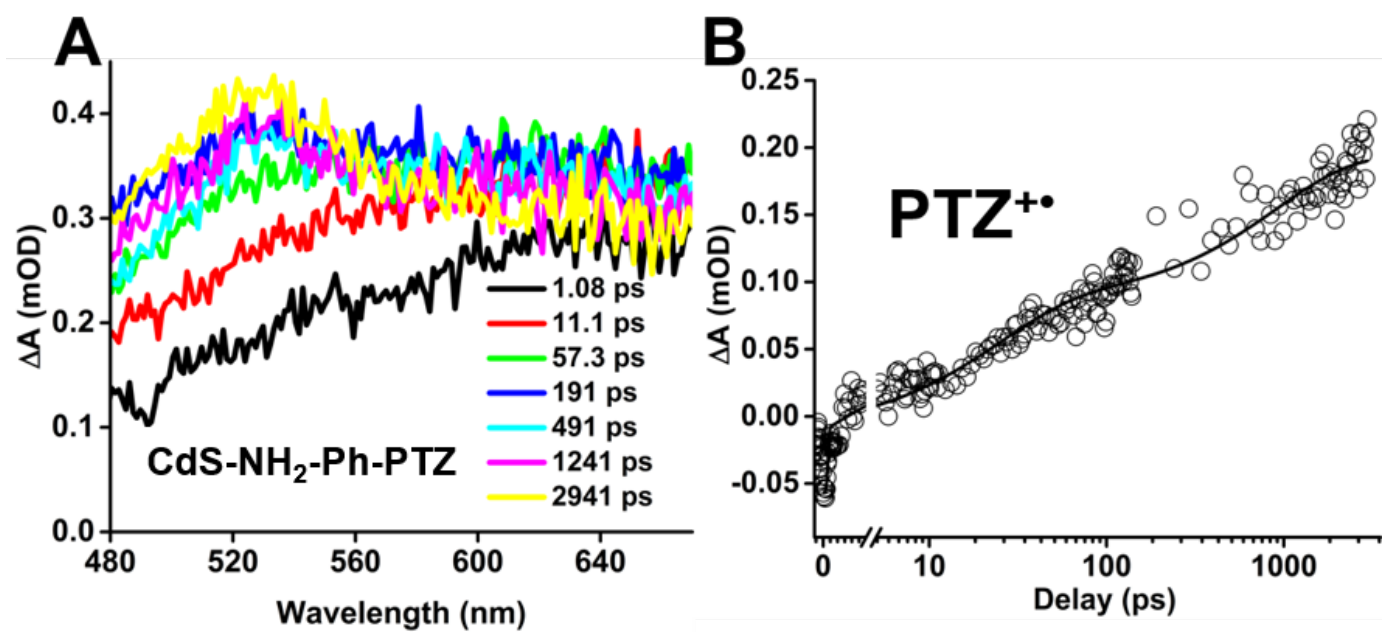

Figure S16. (A) Raw TA spectra of $10 \mu \mathrm{M}$ of CdS QDs with 1000 eq. added PTZ-Ph$\mathrm{NH}_{2}$ at various delay times, after photoexcitation at $420 \mathrm{~nm}$. (B) Plot of deconvoluted kinetic trace at $520 \mathrm{~nm}\left(\mathrm{PTZ}^{+*}\right)$ and the corresponding fit for the same sample as in A. The negative signal in the kinetic trace near time-zero is a nonlinear response of the solvent. Fitting parameters are summarized in Table S4.

Table S4.Time Constants for the Deconvoluted Kinetics Traces Corresponding to $\mathrm{PTZ}^{+\bullet}$ of $10 \mu \mathrm{M}$ of CdS QDs with 1000 eq. added PTZ-Ph- $\mathrm{NH}_{2}$ and Comparison with the Three PTCPTZ Samples Listed in the Main Text ${ }^{a}$

\begin{tabular}{|c|c|c|c|}
\hline Sample & $\begin{array}{c}\text { \# of An-PTZ } \\
\text { per QD } \\
\text { present }^{b}\end{array}$ & $\begin{array}{c}\tau_{1}, \mathrm{ps} \\
\text { ( } \tau_{3} \text { in the main } \\
\text { text) }\end{array}$ & $\begin{array}{c}\tau_{2}, \mathrm{ps} \\
\left(\tau_{4} \text { in the main }\right. \\
\text { text) }\end{array}$ \\
\hline $\begin{array}{l}\text { CdS QDs + } 1000 \\
\text { added An-PTZ }\end{array}$ & 1000 & $23 \pm 2$ & $900 \pm 100$ \\
\hline $\begin{array}{l}\text { CdS-PTC-PTZ with } \\
49 \text { PTC-PTZ bound }\end{array}$ & 33 & $90 \pm 10$ & $2000 \pm 1000$ \\
\hline $\begin{array}{l}\text { CdS-PTC-PTZ with } \\
110 \text { PTC-PTZ bound }\end{array}$ & 66 & $24 \pm 3$ & $700 \pm 100$ \\
\hline $\begin{array}{l}\text { CdS-PTC-PTZ with } \\
130 \text { PTC-PTZ bound }\end{array}$ & 114 & $33 \pm 4$ & - \\
\hline
\end{tabular}

${ }^{a} \tau_{1}$ and $\tau_{2}$ are correspond to $\tau_{3}$ and $\tau_{4}$ in the Table 2 of the main text. ${ }^{b}$ This quantity is measured by ${ }^{1} \mathrm{H}$ NMR signal of PTZ-Ph- $\mathrm{NH}_{2}$ species in the corresponding sample. 
DFT-based Geometry Optimization of Ligands. Quantum chemical calculations were performed at the density functional theory (DFT) level using Nwchem 6.5 software. ${ }^{8}$ Geometries were optimized using the B3LYP functional with an effective core potential basis for the Cd (LANL2DZ) and the 6-31+G** basis set for all other atoms. We performed geometry optimization on Cd(BA-PTZ) 2 and Cd(PTC-PTZ) 2 complexes by using above procedure. The final optimized geometry is in Figure S15. The through-bond distance from the Cd atom to the center of PTZ moiety for Cd(PTC-PTZ) 2 and Cd(BA-PTZ)2 is found to be $12.7 \AA$ and $10.6 \AA$ respectively.

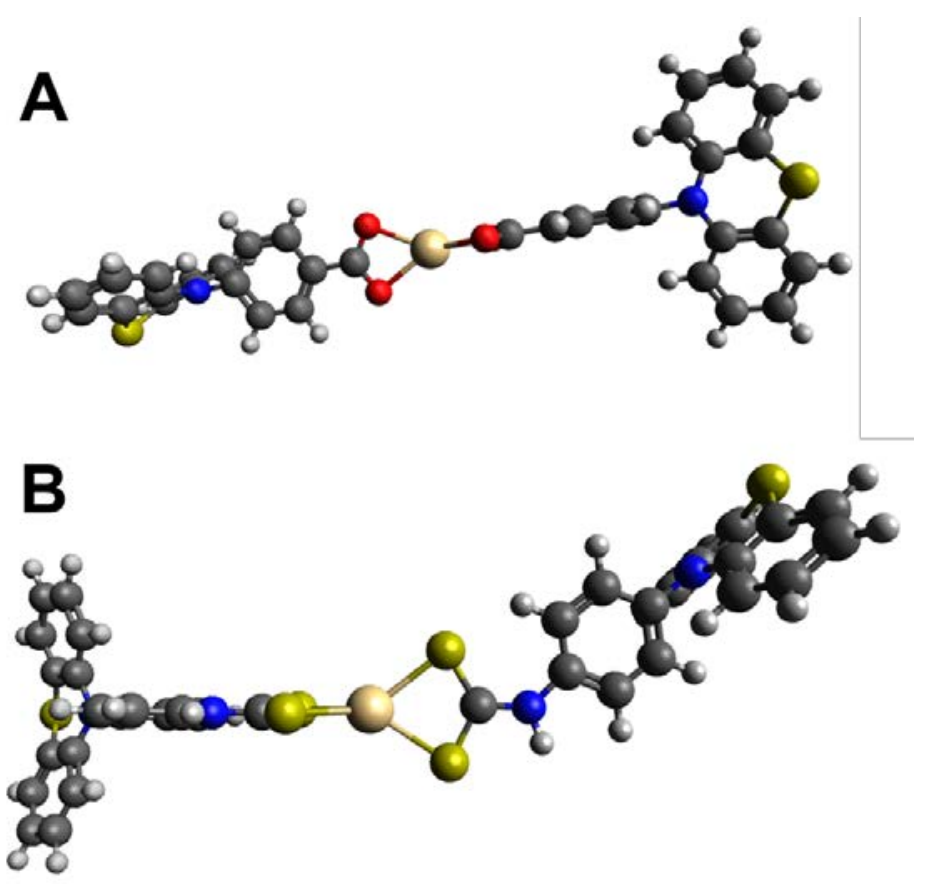

Figure S17. DFT-based geometry optimized (A) Cd(BA-PTZ) 2 and (B) Cd(PTC-PTZ)2. The through-bond distance from the Cd atom to the center of PTZ moiety is found to be $12.7 \AA$ and $10.6 \AA$ respectively.

\section{Femtosecond TA Data for the Mixtures of 10-Phenyl-10H-phenothiazine and CdS QDs.}

In order to verify that the hole transfer process that we described in the main text occurs through the linker molecules (“through-bond”) rather than directly from the QD through via van der Waals interaction with a physisorbed PTZ moiety (“through-space”), we added 1000 eq. of PTZ-Ph, with no linker, to $10 \mu \mathrm{M}$ QDs. Steady-state PL spectra indicate that the PL of the QDs was quenched by 70\%; however we did not observe any formation of $\mathrm{PTZ}^{+\bullet}$ on the picosecond time scale, Figure 
S16. We therefore conclude that the hole transfer processes we report in the main text are "throughbond" hole transfers.
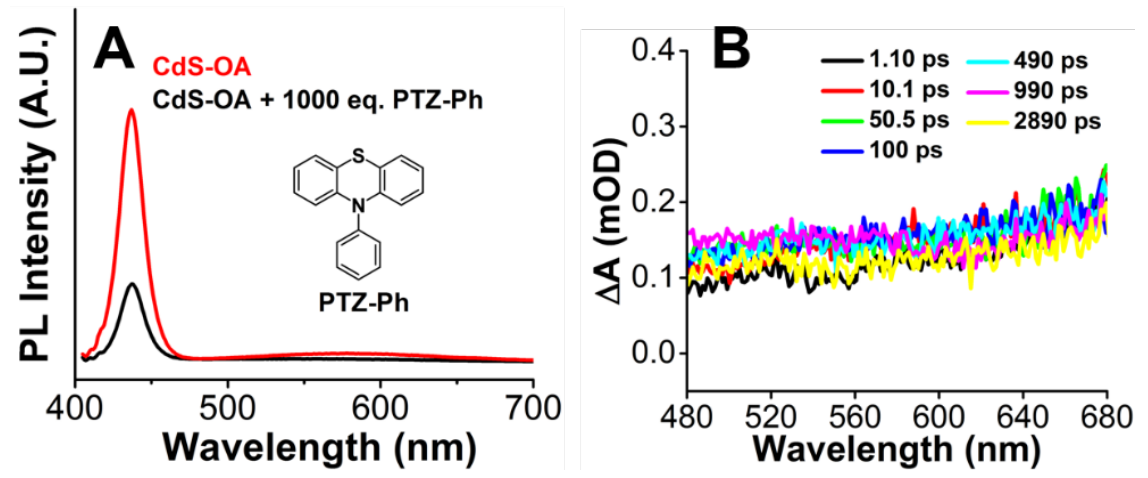

Figure S18. (A) PL spectra of 10 uM CdS QDs coated with OA (red) and CdS-OA QDs treated with 1000 eq. PTZ-Ph per QD (black), excited at $400 \mathrm{~nm}$. (B) TA spectra of CdS-OA with treatment of 1000 eq. PTZ-Ph per QD at various delay time, which shows no formation of PTZ ${ }^{+\bullet}$ $(520 \mathrm{~nm})$ on ultrafast timescale.

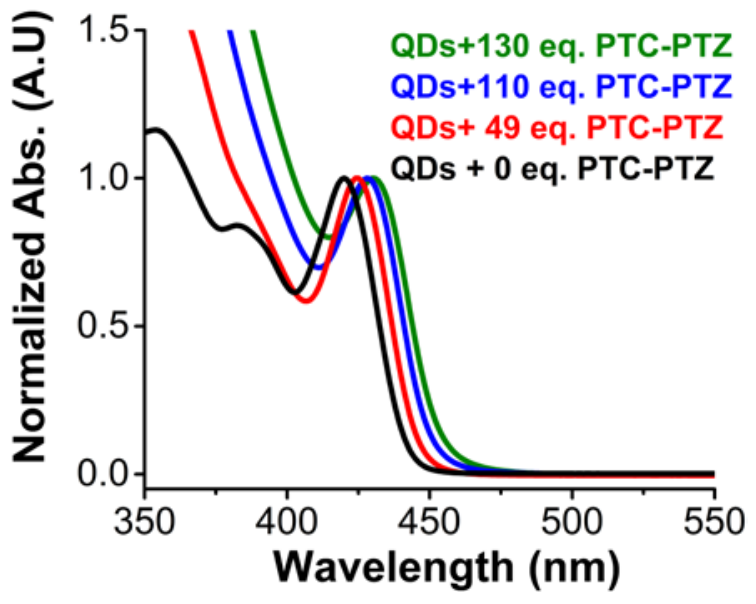

Figure S19. Ground state absorption spectra of CdS QDs with different amount of bound PTC-PTZ as measured by NMR discussed in the main text. 


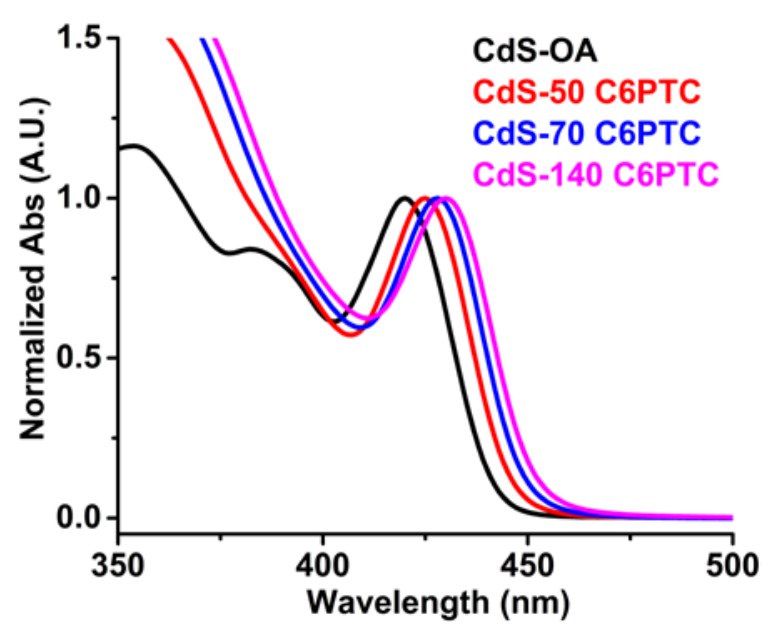

Figure S20. Ground state absorption spectra of CdS QDs with different amount of bound C6PTC as measured by NMR discussed in the main text.

${ }^{1} \mathrm{H}$ NMR Spectra of PTZ-Ph-NH2 and BA-PTZ, Synthesized Based on the Literature Procedure. 9, 10 We synthesized PTZ-Ph- $\mathrm{NH}_{2}$ and BA-PTZ based on previously published procedures. Figure S18 shows the proton NMR spectra match with the NMR spectra described in the literature. 


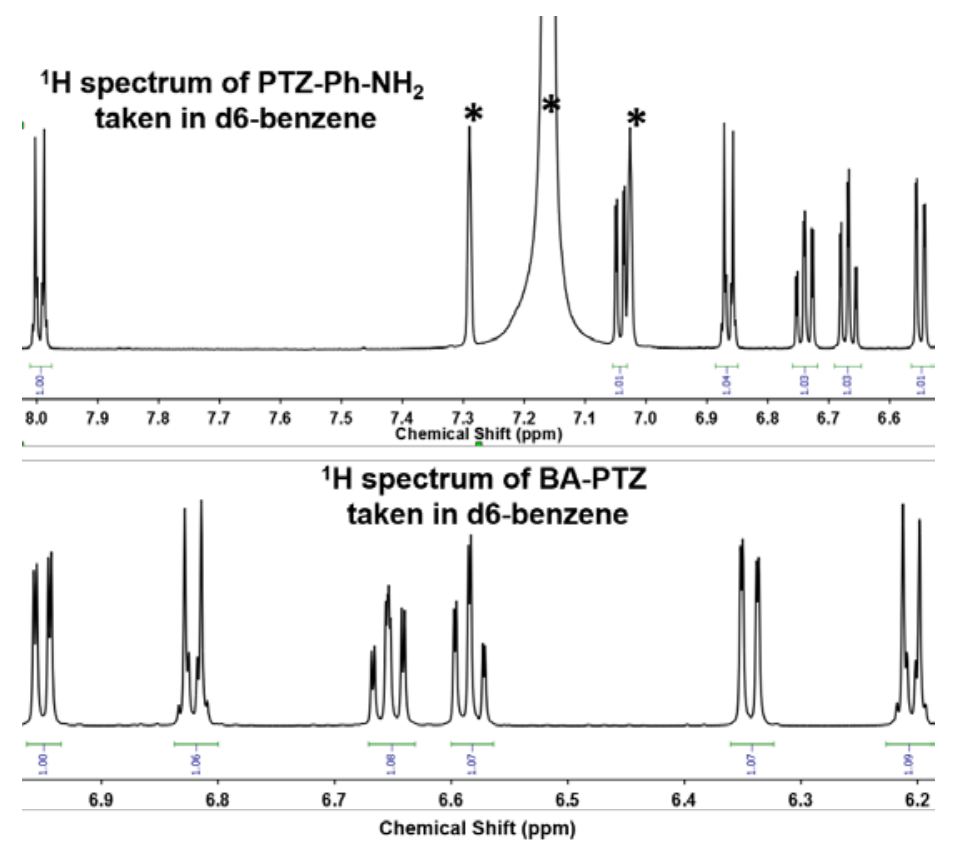

Figure S21. ${ }^{1} \mathrm{H}$ NMR spectra of PTZ-Ph-NH 2 and BA-PTZ synthesized based on the literature procedures. *: signals from solvent d6-benzene

\section{REFERENCES}

1. Yu, W. W.; Qu, L.; Guo, W.; Peng, X. Experimental Determination of the Extinction Coefficient of Cdte, CdSe, and CdS Nanocrystals. Chem. Mater. 2003, 15, 2854-2860.

2. Sahami, S.; Weaver, M. J. Entropic and Enthalpic Contributions to the Solvent Dependence of the Thermodynamics of Transition-Metal Redox Couples: Part I. Couples Containing Aromatic Ligands. J. Electroanal. Chem. Inter. Electrochem. 1981, 122, 155-170. 3. Reiss, H.; Heller, A. The Absolute Potential of the Standard Hydrogen Electrode: A New Estimate. J. Phys. Chem. 1985, 89, 4207-4213.

4. Harris, R. D.; Amin, V. A.; Lau, B.; Weiss, E. A. Role of Interligand Coupling in Determining the Interfacial Electronic Structure of Colloidal CdS Quantum Dots. ACS Nano 2016, 10, 1395-1403.

5. Jin, S.; Harris, R. D.; Lau, B.; Aruda, K. O.; Amin, V. A.; Weiss, E. A. Enhanced Rate of Radiative Decay in CdSe Quantum Dots Upon Adsorption of an Exciton-Delocalizing Ligand. Nano Lett. 2014, 14, 5323-5328.

6. Margulies, E. A.; Wu, Y.-L.; Gawel, P.; Miller, S. A.; Shoer, L. E.; Schaller, R. D.; Diederich, F.; Wasielewski, M. R. Sub-Picosecond Singlet Exciton Fission in Cyano-Substituted Diaryltetracenes. Angew. Chem., Int. Ed. 2015, 54, 8679-8683.

7. McArthur, E. A.; Morris-Cohen, A. J.; Knowles, K. E.; Weiss, E. A. Charge Carrier Resolved Relaxation of the First Excitonic State in CdSe Quantum Dots Probed with NearInfrared Transient Absorption Spectroscopy. J. Phys. Chem. B 2010, 114, 14514-14520.

8. $\quad$ Valiev, M.; Bylaska, E. J.; Govind, N.; Kowalski, K.; Straatsma, T. P.; Van Dam, H. J. J.; Wang, D.; Nieplocha, J.; Apra, E.; Windus, T. L.; de Jong, W. A. Nwchem: A Comprehensive and Scalable Open-Source Solution for Large Scale Molecular Simulations. Comput. Phys. Commun. 2010, 181, 1477-1489. 
9. Weiss, E. A.; Ahrens, M. J.; Sinks, L. E.; Gusev, A. V.; Ratner, M. A.; Wasielewski, M. R. Making a Molecular Wire: Charge and Spin Transport through Para-Phenylene Oligomers. $J$. Am. Chem. Soc. 2004, 126, 5577-5584.

10. Park, Y. S.; Choi, J.; Kim, D.; Lee, B.; Suh, H.; Kim, J. H. Synthesis, Optical, and Electroluminescent Properties of Alternating Copolymer Based on Phenothiazine and Fluorene with Oxadiazole Pendant. Mol. Cryst. Liq. Cryst. 2011, 550, 294-303. 